\title{
Beiträge zur Kenntniss der Eiweisskörper der Kuhmilch.
}

Von

\section{Dr. Schmidt-Muilheim.}

\section{Einleitung.}

Bereits seit längerer Zeit hat man erkannt, dass nach dem Ausfällen des Caseïns und Albumins noch eiweissartige Substanzen in der Milch enthalten sind, die sich von den genannten Körpern in wesentlichen Reaktionen unterscheiden. Auch fehlt es derartigen Substanzen nicht an Namen: Millon und Comaille sprechen von Lactoproteïn, Bouchardat und Quevenne von Albuminose, Morin von Galactin.

Nach neneren Untersuchungen sollen die genannten Körper mit Pepton identisch sein und man ist geneigt gewesen, das Auftreten desselben der Behandlungsweise der Milch (Einwirkung von Säuren, Kochen etc.) zuzuschreiben (Ssubotin).

Hoffmeister behauptet, dass die Milch bis zum Eintritte der Gerinnung kein Pepton enthalte, und dass dieses um so reichlicher anzutreffen sei, je länger die sauer gewordene Milch vorher gestanden habe.

Arnold macht ähnliche Angaben, obne indessen die Gegenwart von Pepton an den Gerinnungsvorgang zu knüpfen; er sagt, dass in frischer Milch kein Pepton angetroffen werde, wohl aber in solcher, die 10-20 Stunden gestanden, wobei eine fortwährende Zunahme an dieser Substanz zu konstatiren sei.

Nach Kirehner jedoch ist Pepton schon in der Milch präformirt enthalten, diese Thatsache sei für die Ernährung sehr wichtig, denn eine an Pepton reiche Milch besitze höheren Nährwerth als eine daran arme. 


\section{Kritik der bisherigen Untersuchungsmethoden.}

Unterwirft man die von den Genannten in Anwendung gezogenen Methoden einer näheren Prifung, so wird der zwischen ihren Angaben bestehende Widerspruch sofort erklärlich, und es findet sich weiter, dass keins von den bisher in Anwendung gebrachten Verfahren den an eine brauchbare Methode zu stellenden Anforderungen auch nur annähernd genügt.

Am auffallendsten tritt uns dieses bei Arnold entgegen. Ihm ist es unbekannt, dass eine spezifische Peptonreaktion bis zur Stunde nicht existirt, und dass die Biuretreaktion den Eiweisskörpern so gut zukommt wie dem Pepton. Seine Angaben sind schon aus dem Grunde werthlos, weil er bei Gegenwart des ganzen Albumins mittels der blossen Biuretreaktion auf Pepton gepriff hat.

Anch Kirchner spricht in dem gleichen Sinne von einer charakteristischen Peptonprobe, sucht aber wenigstens vor der Prüfung nicht allein das Caseïn, sondern auch das Albumin zu entfernen. Nur ist die von ihm benutzte Methode (Zufitgen von soviel Alkohol, dass nur Caseỉn und Albumin, nicht aber auch Pepton gefällt wird) praktisch kaum ausführbar. Ausserdem wird bei diesem Verfahren die Biuretreaktion, wie wir gleich näher sehen werden, durch die Gegenwart des Milehzuckers ganz ausserordentlich beeinträchtigt.

Hoffme ister endlich fällt zunächst mit Essigsäure und sucht den Rest der Eiweisskörper durch Kochen bei Gegenwart von Bleioxyd und etwas essigsaurem Blei zu entfernen. Hat er sich alsdann mittels Essigsäure und Bluṭlangensalz von der völligen Abwesenheit des Caseïns and Albumins uiberzeugt, so benutzt er nunmehr zum Nachweise des Peptons die Biuretreaktion. Hoffmeister hat es unterlassen, sich von der Zuverlässigkeit dieser Methode zu überzeugen. Ihr haften, wie ich fand, selr erhebliche Mängel an.

Zunächst nämlich fällen die in Anwendung gezogenen Reagentien nicht allein Caseïn und Albumin, sondern sie entziehen auch eine sehr erhebliche Menge von Pepton dem Nachweise. Folgende Versuche beweisen das:

Versuch I. 50 cc frisch centrifugirter Milch, welche, wie ich nach einem anderen Verfahren ermittelte, einen Peptongehalt von mehr als $0,1 \%$ besass, werden mit $50 \mathrm{mgr}$ eines ganz reinen Peptons in Lösung versetzt. Das Caseïn wird nach dem Hoppe-Seyler'schen Verfahren entfernt und es 
wird nunmehr die Flüssigkeit mit Bleioxydhydrat und etwas essigsaurem Blei gekocht. Nach dem Erkalten wird der Ueberschuss an Blei durch einen Schwefelwasserstoffstrom entfernt und das Filtrat auf ein kleines Volumen gebracht. Die stark gelb gefärbte Flïssigkeit, welche c. 20 ce misst, wird nach dem Ansäuern (Salzsäure) mit Phosphorwolframsäure versetzt. Es entsteht kein Niederschlag, wohl aber bildet sich ein solcher auf Zufügen von Spuren einer Peptonlösung sofort.

Das Pepton hat sich mithin dem Nachweise entzogen.

Versuch II. 50 cc Milch werden mit $90 \mathrm{mgr}$ Pepton versetzt und dann wie oben behandeit. In dem stark eingeengten, entbleiten und mit Salzsäure versetzten Filtrate entsteht auf Zufügen von Phosphorwolframsäure ein schwacher flockiger Niederschlag. Derselbe wird mit stark verdünnter Salzä̆ure gewaschen und in Natronlauge gelöst (näheres über dieses Verfahren s. hinten). Auf Zusatz von Kupfersulphat zeigt sich nur eine Andeutung einer Biuretreaktion, trotzdem die Flüssigkeit nur ein Volumen von $9 \mathrm{cc}$ einnimmt.

Es hat sich daher auch in diesem Falle das Pepton so gut wie vollständig dem Nachweise entzogen.

Sodann ist es aber auch ganz und gar unstatthaft, eine Peptonbestimmung bei. Gegenwart von Milchzucker vorzunehmen, weil diesem, wie die folgenden Versuche beweisen, im hohen Grade die Fähigkeit innewohnt, die Biuretreaktion zu beeinträchtigen:

Versuch III. Eine fünfprozentige Milehzuckerlösung wird mit soviel Pepton versetzt, dass der Gehalt an dieser Substanz $0,1 \%$ ausmacht. Beim Zufügen von Natronlauge und Kupfersulphat nimmt die Flüssigkeit einen schwachen schmutzig-röthlichen Farbenton an, der nach kurzer Zeit mehr in's Gelbliche übergeht. Eine reine Peptonlösung von gleicher Konzentration zeigt unter denselben Verhältnissen eine intensive Rothfärbung, gegen welche die mit Kupfer versetzte zuckerhaltige Peptonlösung fast völlig farblos erscheint.

Um nụn weiter zu erfahren, in welchem Umfange die Biuretreaktion in der Milch durch den Zucker verdeckt werden kann, habe ich abgemessene Quantitäten einer Peptonlösung von genau bekanntem Gehalte mit soviel Milchzucker versetzt, dass die Fliissigkeit im Gehalte an diesen Substanzen annähernd mit der normalen Milch übereinstimmte und alsdann die quantitative Bestimmung des Peptons nach einem frïher von mir angegebenen Verfahren ${ }^{1}$ ) bewirkt.

1) Die Thatsache, dass eine von mir in Vorschlag gebrachte Methode zur quantitativen Bestimmung des Peptons vielfach (z. B. unlängst noch von 
Versuch IV. 10 ce einer 10prozentigen Milchzuckerlösung werden mit 10 mgr Pepton versetzt. Nach Zusatz von Natronlauge und Kupfersulphat

Schulze und Barbieri) als Hoffmeister's Verfahren bezeichnet wird, gibt mir zu folgenden Bemerkungen Anlass:

Im Beginn des Jahres 1880 beschrieb ich in du Bois-Reymond's Archiv für Physiologie p. 34 und 35 folgendes Untersuchungsverfahren:

„Um zu einer quantitativen Bestimmung des Peptons zu gelangen, smachte ich von der Eigenschaft desselben Gebrauch, auf Zusatz von Natron"lauge und Kupfersulphat eine schöne weinrothe Färbung anzunehmen, die "um so intensiver hervortritt, je mehr Pepton vorhanden ist. Nachdem er"mittelt war, dass Lösungen von 1 Theil Pepton in 2000 bis 3000 Theilen "Wasser die vortheilhaftesten Konzentrationen für die kolorimetrische Me"thode abgaben, wurde eine Normalpeptonlösung auf die Weise bereitet, "dass man ein abgewogenes Quantum Pepton in Wasser löste, mit Natron"lauge versetzte und jetzt so lange eine sehr verdünte Kupfersulphatlösung „zufügte, bis die anfänglich weinrothe Färbung der Flüssigkeit eben erkenn"bar in's Blaue zu schimmern begann. War dieser Punkt erreicht, so gab "man dem Gemenge durch Zufügen von Wasser eine derartige Konzentration, „dass $3000 \mathrm{cc}$ Flüssigkeit $1 \mathrm{gr}$ Pepton enthielten. Sollte nun eine Pepton"bestimmung stattfinden, so wurde die zu untersuchende Flüssigkeit, nachdem "man sie vorher von allen wahren Eiweisskörpern befreit hatte, mit einem "kleinen Quantum Natronlauge versetzt und dann in ähnlicher Weise mit "Kupfersulphat behandelt wie die Normallösung. Hatte auch sie einen eben "bemerkbaren Ton in's Blaue angenommen, so bestimmte man ihr Volumen "und es wurden jetzt abgemessene Mengen der Normallösung sowohl als der „)anderen Flüssigkeit in parallelwandige Glaströge gebracht, wie sie schon "seit lange zur Bestimmung der Färbekraft des Blutes benutzt werden. Die "Wandungen aller von mir benutzten Tröge waren genau $2 \mathrm{~cm}$ von ein"ander entfernt. Diese Gläser stellte man einem Fenster gegenüber auf "einen Tisch und versah sie mit einem weissen Hintergrunde. Ueber den "unmittelbar neben einander stehenden Trögen befanden sich mit Wasser „gefüllte Büretten; aus diesen liess man unter stetigem Umrühren der Flüs„sigkeiten mit einem Glasstabe so lange Wasser in die Gläser treten, bis "beide Lösungen in ihren Farbentönen genau übereinstimmten. Da der Pep"tongehalt der Normallösung bekannt war, so liess sich jetzt auf sehr ein"fachem Wege die Menge des in der anderen Flüssigkeit enthaltenen Pep„tons ermitteln.

"In der Absicht, den. Fehler aufzufinden, dem mein Auge bei der Her"stellung der Farbengleichung ausgesetzt war, liess ich mir von befreundeter "Hand eine Reihe von Lösung'en mit bekanntem Peptongehalt bexeiten and "bestimmte darauf diesen letzteren kolorimetrisch.

"Dabei ergaben sich die folgenden Zahlen, zu deren Würdigung be- 
wird die Flüssigkeit auf 20 cc gebracht. Bei dieser Konzentration stimmt ihr violetter Farbenton etwa mit der Biuretreaktion einer Normallösung von $1: 6000$ iberein.

Anstatt 0,01 Pepton daher nur 0,0034 angezeigt.

"merkt werden muss, dass sie aus einer Zeit stammen, in welcher ich in der „Vergleichung der Farben noch wenig geïbt war.

I. Reihe.

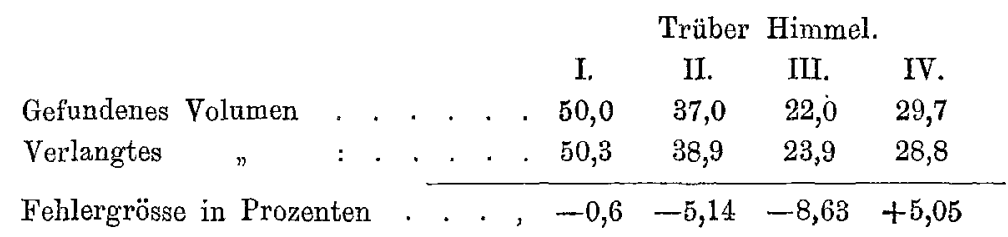

II. Reihe.

\begin{tabular}{|c|c|c|c|c|c|c|c|}
\hline & & & I. & II. & III. & IV. & $\mathrm{V}$. \\
\hline Gefundenes & Volumen & . & 41,5 & 38,2 & 84,0 & 34,6 & 23,0 \\
\hline Verlangtes & $n$ & . & 38,7 & 39,6 & 35,1 & 34,2 & 21,6 \\
\hline Versuchsfeh & in $\operatorname{Pr}$ & ten & $+6,8$ & $-3,66$ & $-3,23$ & $+1,16$ & $+6,09$ \\
\hline
\end{tabular}

III. Reihe.

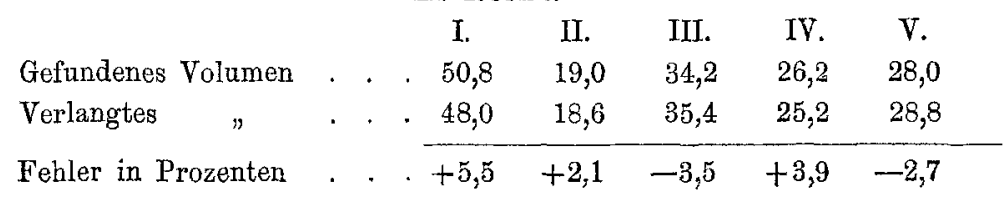

"Abgesehen von einer einzigen aus der Reihe fallenden Beobachtung "schwanken also äussersten Falls die Fehler zwischen \pm 6 Prozent, in der "Regel aber liegen sie in weit engeren Grenzen.

Viele Monate später sehrieb Hoffmeister:

"Hin und wieder habe ich mich zur Peptonbestimmung statt der po"larimetrischen Methode eines kolorimetrischen Verfahrens bedient, ähnlich je"nem, welches Schmidt-Mülheim in Anwendung gezogen bat. Dasselbe be"ruht auf der Fähigkeit der Peptonlösungen, mit Kupfer und Natronlauge "violette Flüssigkeiten zu bilden, deren Färbekraft, passenden Kupferzusatz "vorausgesetzt, proportional dem Peptongehalte ist.

„Durch Kupfer und Natronzusatz zu Peptonlösungen von genau bekann"tem Gehalte stellte ich mir eine Art Farbenskala her, mit der die auf "Pepton zu untersuchenden Flüssigkeiten nach Zusatz von Kupfervitriol und "Natronlauge bis zu einer bestimmten rothvioletten Nuance in gleich dicken "Schichten verglichen wurden."

Bis auf die kleine Abweichung, dass $\mathrm{H}$ offmeister nicht eine Normallösung von fest bestimmtem Peptongehalte, sondern mehrere derartige Lö- 
Versuch V. 10 co derselben Milchzuckerlösung mit 0,02 Pepton versetzt und dann in ähnlicher Weise behandelt. Die Flüssigkeit stimmt bei 20 cc mit einer Normallösung von 1: 4000 überein.

Anstatt 0,02 däher nur 0,005 gr Pepton gefunden.

Durch die vorstehenden Zahlen wird indessen noch nicht die ganze Mangelhaftigkeit der von $\mathrm{H}$ offmeister benutzten Methode ausgedrückt. Die Milch nämlich verfügt noch ïber einen gelben Farbstoff, der sehr wohl befähigt ist, den schwachen Farbenton in Zucker-Peptonlösungen von vorstehender Konzentration so gut wie vollständig zu verdecken. Man darf daher wohl mit Recht behaupten, dass ein $0,1-0,2$ prozentiger Peptongebalt der Milch sich bei Anwendung des Hoffmeister'schen Verfahrens dem Nachweise gänzlich entziehen würde.

\section{Neues Untersuehungsverfahren.}

Aus dem Vorstehenden exgiebt sich, dass der Frage nach dem Peptongehalt der Milch nur dann mit Aussicht auf Erfolg näher getreten werden kann, wenn es gelingt, das Pepton von den Eiweisskörpern der Milch scharf zu trennen und von den schädlichen Beimengungen des Zuckers und des Farbstoffes zu befreien.

Der ersten dieser Aufgaben glaubte ich anfänglich durch ein frither von mir beschriebenes und bei der Untersuchung des Blutes sowie des Magen-Darminhaltes mit trefflichstem Erfolge benutztes Verfahren-Ausfällen des Eiweisses mittels essigs. Eisenoxyd in der Siedhitze und bei ganz schwach saurer Reaktion der Fliissigkeit - gerecht werden zu können. Indessen erwies sich diese Methode deshalb nicht als tadelfrei, weil der Milchzucker leicht einiges Eisen in Lösung hält.

Weit weniger geeignet noch zeigte sich die Methode Ritt-

sungen von verschiedener Konzentration benutzt, ist also das von ihm in Anwendung gebrachte Verfahren zur quantitativen Bestimmung des Peptons, dessen Zuverlässigkeit zu prüfen er übrigens für ganz überflüssig hält, eine ganz genaue Kopie des meinigen. Die winzige von Hoffmeiste $\mathbf{r}$ herrührende Abänderung aber kann ich nicht als eine Verbesserung betrachten, denn nicht ohne Grund habe ich angegeben, dass Lösungen von 1 Theil Pepton in ea. 2000 bis 3000 Theilen. Wasser die vortheilhaftesten Konzentrationen abgeben. 
hausen und zwar deshalb, weil sie bis auf meistens nur sehr geringe Reste das Pepton dem Nachweise entzieht.

Brauchbarkeit bekundete nun ein älteres Verfahren, auf welches Salkowski neuerdings wieder die Aufmerksamkeit gelenkt hat. Es beruht darauf, die Flissigkeiten zunächst mit Kochsalz zu versetzen und dann ein entsprechendes Volumen einer mit Essigæäure gemischten, gesättigten Kochsalzlösung zuzufügen. Die erwähnten Reagentien fällen die Eiweisskörper völlig aus, während sie in reinen Peptonlösungen anch nicht Spuren einer Trïbung hervorrufen, was ich deshalb besonders hervorhebe, weil es ja nicht an Stimmen gefehlt hat, welche diese Reagentien zu den Fällungsmitteln für Pepton gezählt haben.

Diese Methode nun sehien mir um so grössere Vortheile darzubieten, als bei ihrer Anwendung eine Entstehung des Peptons in Folge der Behandlungsweise völlig ausgeschlossen ist. Weit eher als an eine Bildung ist unter diesen Verhältnissen an eine Umformung des Körpers zu denken, denn Henninger und Hoffmeister haben uns ja gezeigt, dass kräftige wasserentziehende Einflïsse das Pepton in gerinnbares Eiweiss überzuführen begabt sind.

Dass die genannten Reagentien die kolorimetrische Bestimmung des Peptons thatsachlich nicht beeinträchtigen, ergiebt sich aus folgendem Versuche, dem ich noch mehrere andere mit gleichem Erfolge an die Seite stellen könnte.

Versuch VI. 20 ce einer $1 \% \%$ Peptonlösung werden mit Kochsalz gesättigt und alsdann mit $50 \mathrm{cc}$ einer aus 1 Vol. Eisessig und 5 Vol. konz. Kochsalzlösung bestehenden Mischung versetzt. Nunmehr fällt man das Pepton mittels Phosphorwolframsäure, sammelt den Niederschlag und löst ihn nach dem Auswaschen mit verdünnter Salzsäure in Natroulauge. Die Lösung nimmt auf Zusatz von Kupfer einen blauen Farbenton an, der indessen nach 1/2 stündigem Stehen einem reinen Weinroth weicht. Gleichzeitig finden sich anf dem Boden des Glases geringe Mengen eines von der Zersetzung dex Phosphorwolframsäure herrührenden Niederschlages, die durch Filtration beseitigt werden.

Bei der nun erfolgenden kolorimetrischen Peptonbestimmung beträgt die Konzentration der Normallösung $1: 2000$. Der gelöste Phosphorwolframniederschlag nimmt ein Volumen von $38 \mathrm{cc}$ ein, als sein Farbenton mit dem der Normallösung übereinstimmt. Die kleine Differenz (verlangt werden $40 \mathrm{ce})$ liegt innerhalb der Fehlergrenzen der Methode.

Hatte sich somit die Brauchbarkeit des Verfahrens reinen 
Peptonlösungen gegenüber bewährt, so war es nunmehr in seiner Anwendung auf Milch einer Prïfung zu unterziehen. Zu dem Ende wurde zunächst Milch für sich und sodann nach Zusatz einer bestimmten Peptonmenge auf ihren Peptongehalt untersucht.

Versuch VII. 40 ec Milch werden mit 40 ec Wasser verdünnt. Alsdann wird das Gemisch mit Kochsalz gesättigt und mit $70 \mathrm{cc}$ der EssigsäureKochsalzlösung versehen. 75 cc des klaren Filtrats, welches auf Zufügen von Blutlaugensalz auch nicht Spuren einer Trübung erkennen lässt, werden mil Phosphorwolframsäure versetzt. Der entstandene flockige Niederschlag wird aufgesammelt und wie oben behandelt.

Der gelöste Niederschlag nimmt bei Uebereinstimmung mit einer Normallösung von $1: 2000$ ein Volumen von 58 ce ein. Die Milch würde demnach einen Peptongehalt von $0,145 \%$ besitzen.

Versuch VIII. 40 ce derselben Milch werden mit 40 ce einer $1 \% \%$ Peptonlösung versetzt und dann genau in der gleichen Weise beliandelt.

Der in Natronlauge gelöste und mit einer genügenden Kupfermenge behandelte Niederschlag nimmt bei Uebereinstimmung mit einer Normallösung von 1:2000 ein Volumen von 184 ce ein, während nach dem Ausfall des vorigen Versuches 196 ec zu erwarten waren.

Weiter galt es, den durch die Gegenwart des Milchzuckers und des Farbstoffes bedingten Versuchsfehlern zu entgehen und ich bediente mich hierzu eines Verfahrens, welches mit einer von Hoffmeister empfohlenen Methode ${ }^{1}$ ) im Prinzip ibereinstimmt. Die von ibrem Caseïn und Albumin befreite Milch wird mit Phosphorwolframsäure versetzt, der hierdurch bewirkte Niederschlag auf dem Filter gesammelt, mit verdünnter Salzsäure ausgewaschen und nunmehr, ohne vom Filter genommen zu werden, in Natronlange gelöst. Zu letzterer Operation diente eine mit Natron beschickte Spritzflasche. Reduktionsvorgänge bewirkten nicht selten eine m. o. w. nennenswerthe Blaufärbung der Lösung. Diese versehwand indessen schon nach kurzer Zeit und es liess nunmehr

1) Hoffmeister (chem. Centralbl. Jahrg. 1880) bringt den Phosphorwolframsäureniederschlag auf ein Filter und wäscht ihn mit Schwefelsäure. Der Filterinhalt wird alsdann in eine Schale gebracht, mit festem Barythydrat und Wasser vorrieben und kurze Zeit erwärmt. Zn der von den unlöslichen Baryumverbind'nngen abfiltrirten barythaltigen Flüssigkeit fügt man direkt einige Tropfen Kupferlösung; das Auftreten einer rothen oder violetten Färbung zeigt die Anwesenheit von Pepton an. 
die Lösung auf behutsamen Zusatz von Kupfervitriol die Biuretreaktion in einem überaus reinen Farbentone hervortreten.

Die Anwendbarkeit dieses Verfahrens ergab sich aus folgenden Versuchen:

Versuch IX. 20 cc einer $5 \%$ Milchzuckerlösung werden mit $20 \mathrm{mgr}$ Pepton versetzt und mit Salzsäure angesäuert. Nunmehr wird das Pepton mittels Phosphorwolframsäure gefällt und der Niederschlag genau nach den oben gegebenen Vorschriften behandelt.

Die Bestimmung wird doppelt ausgeführt. Normallösung $1: 1000$.

Bestimmung a.

Volumen der Flïssigkeit bei Uebereinstimmung mit der Normallösung $18,5 \mathrm{cc}=18,5 \mathrm{mgr}$ Pepton.

Bestimmung b.

Volumen der Flüssigkeit bei Uebereinstimmung mit der Normallösung $19 \mathrm{cc}=19 \mathrm{mgr}$ Pepton.

Versuch X. Von einer Peptonlösung, deren Gehalt nicht näher bestimmt ist, werden 10 cc mit Phosphorwolframsäure gefällt, nachdem sie zuvor mit Salzsäure versetzt worden. Die weitere Behandlung erfolgt genau wie oben.

Es werden zwei derartige Bestimmungen ausgeführt. Die Normallösung wird bereitet, indem man 10 ec derselben Peptonlösung mit Natronlauge und einer genügenden Menge Kupfervitriol versetzt und nunmehr das Ganze auf 20 cc bringt.

Bestimmung a.

Bei 19 cc Uebereinstimmung mit der Normallösung. Versuchsfehler daher $-5 \%$.

Bestimm ung b.

Bei 18,5 cc Uebereinstimmung mit der Normallösung. Versuchsfehler daher $-7,5 \%$

\section{Die eiweissartigen Körper der Milch und ihr Verhalten beim Digeriren.}

Darf man die beschriebene Methode als zuverlässig anerkennen, so kann man sich jetzt sehr leicht ein Urtheil darüber bilden, wie weit bei den gebränchlichen Methoden zur Ausfällung des Caseïns und Albumins von einem Auftreten des Peptons in Folge der Behandlungsweise der Milch die Rede sein kann. Im Nachfolgenden sind zahlreiche derartige vergleichende Peptonbestimmungen mitgetheilt und es ergiebt sich daraus, dass bei Anwendung des 
Hoppe-Seyler'schen Verfahrens eine Peptonisirung in Folge der Linwirkung der Säure oder des Kochens nicht nachzuweisen ist und dass also diese Methode, deren Zuverlässigkeit hinsichtlich der Caseïn- und Albuminwerthe ich an einer anderen Stelle (s. d. Arch. p. 243) hervorgehoben habe, auch eine genaue Bestimmung des Peptons gestattet.

Am letztgenannten Orte wurde auch gezeigt, dass die Caseïnmenge keineswegs einen constanten Werth besitzt, sondern dass sie von der mehr oder weniger frischen Beschaffenheit des Drüsensekretes abhängig ist, und zwar so, dass das Caseïn proportional der Dauer des Stehens bei gewöhnlicher Temperatur oder des Digerirens bei Körperwärme an Menge einbüsst. Da sich nun weiter fand, dass ganz frische Milch einen mässigen Peptongehalt besitzt, der durch ein Digeriren der Milch bei $40^{\circ}$ oder durch längeres Stehenlassen bei Zimmerwärme derartig anwachsen kann, dass er dem Albumingehalt, der durch die genannten Eingriffe keine Veränderung erleidet, an Grösse kaum noch nachsteht, so musste es nahe liegen, Caseïnabnahme und Peptonzuwachs in einen ursächlichen Zusammenhang zu bringen, und das um so mehr, als ein oberflächlicher Vergleich ergab, dass die Caseïnmenge einigermassen im Umfange des Peptonzuwachses schwindet.

Die nachfolgenden Versuche gewähren einen näheren Einblick in diese Verbältnisse:

Versuch XI. 5/X 81. Morgenmilch, spez. Gew. 1,0307. Sowohl in der frischen als in der 10 Stunden hindurch bei Körperwärme digerirten Milch finden Peptonbestimmungen wie auch Bestimmungen der sämmtlichen Eiweisskörper nach dem modificirten $\mathrm{H}$ oppe-Seyler'schen Verfahren statt.

\section{A. frische Milch.}

1) Direkte Peptonbestimmungen.

Je 20 cc Milch werden mit 20 cc Wasser verdünnt. Das Gemisch wird mit Kochsalz gesättigt. Nunmehr werden 40 co der Essigsäure-Kochsalzmischung zugefügt. Die Niederschläge werden gesammelt und mit dem gleichen Gemisch gewaschen. In den klaren Filtraten wird das Pepton mittels Phosphorwolframsäure gefällt. Die weitere Behandlung der Niederschläge erfolgt nach den oben angegebenen Vorschriften.

Der Inhalt von Glas a nimmt ein Volumen von $25 \mathrm{cc}$ ein als sein Farbenton mit demjenigen einer $1 \% \%$ Normalpeptonlösung ẗbereinstimmt; der Inhalt von Glas b misst unter denselben Verhältnissen $27 \mathrm{cc}$. 
Glas a zeigt daher einen Peptongehalt von $\mathbf{0 , 1 2} \%$ an.

"b " " " $" \quad, \mathbf{0 , 1 3} \%$ "

2) Eiweiss- und Peptonbestimmungen nach dem modificirten Hoppe-Seyler'schen Verfahren.

Die Bestimmungen des Caseïns und Albumins finden genau nach den in einer früheren Arbeit angegebenen Vorschriften statt. In den eingeengten caseïn- und albuminfreien Filtraten wird sodann das Pepton mittels Phosphorwolframsäure niedergeschlagen, auf dem Filter gesammelt, gehörig mit verdïnnter Salzsäure gewaschen und nun in der oben angegebenen Weise weiter behandelt.

a) Caseinbestimmung.

Je 20 ec Milch mit 400 ce Wasser verdünnnt. Ein Zusatz von 11,5 cc der sehr verdünnten Essigsäure sowie ein nachfolgender stärker Kohlensäurestrom genügen, das Caseïn gut zur Ausscheidung zu bringen. Die Niederschläge werden auf dem Filter mit Alkohol entwässerrt und mittels Aether entfettet.

\begin{tabular}{|c|c|c|c|}
\hline Filter und & Caseïn & $\begin{array}{c}\text { Best. I. } \\
49,936\end{array}$ & $\begin{array}{c}\text { Best, II. } \\
49,914\end{array}$ \\
\hline Filter. & . . . & 49,443 & 49,423 \\
\hline & Caseïn & 0,493 & 0,491 \\
\hline
\end{tabular}

b) Albuminbestimmung.

Die Filtrate werden zum Sieden erhitzt und dann zur besseren Ausscheidung des Albumins noch mit 5 co verdünnter Essigsäure versetzt. Nach dem Aufsammeln der hierbei entstandenen Niederschläge werden die Flüssigkeiten auf 25 cc eingeengt. Hierbei fallen nur sehr unerhebliche Flocken aus, die mit den bereits gewonnenen Niederschlägen vereint werden. Die Niederschläge und Filter werden sodann tüchtig ausgewaschen und getrocknet.

Best. I. Best II.

Filter und Albumin. . 49,472 49,535

Filter . . . . . . . $49,389 \quad 49,456$

$$
\begin{array}{rcc}
\text { Albumin } & 0,083 & 0,079 \\
=\mathbf{0}, 40 \% & =\mathbf{0 , 3 8} \% .
\end{array}
$$

c. Peptonbestimmung.

In den caseïn- und albuminfreien Filtraten wird das Pepton mittels Phosphorwolframsäure niedergeschlagen und nach dem oben angegebene Verfahren kolorimetrisch bestimmt. Konzentration der Normalpeptonlösung $1: 1000$.

Glas a zeigt bei 24 co Uebereinstimmung mit der Normallösung, Glas b bei $26 \mathrm{cc}$. 
a zeigt somit einen Peptongehalt von $\mathbf{0 , 1 1} \%$ an.

b " " " " $" 0,12 \% "$

Beide Methoden der Peptonbestimmung liefern also gut übereinstimmende Werthe.

\section{B. Dieselbe Milch nach 10stïndigem Digeriren bei $40^{\circ}$.}

1) Direkte Peptonbestimmung.

Verfahren wie bei der frischen Milch. Normallösung 1: 1000.

Inhalt von Glas a stimmt bei $62 \mathrm{cc}$ im Farbenton mit der Normallösung überein.

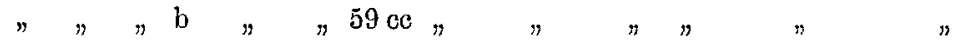

Glas a zeigt daher einen Peptongehalt von $\mathbf{0 , 3 0 \%} \%$, Glas $b$ einen solchen von $0,29 \%$ an.

2) Eiweiss- und Peptonbestimmungen nach dem modif.

Hoppe-Seyler'schen Verfahren.

a) Caseinbestimmung.

Best. I. Best. II.

Filter und Caseïn . . 49,931 49,894

Filter . . . . . . . $49,480 \quad 49,444$

Casein $0,451 \quad 0,450$

$$
=\mathbf{2 , 1 9} \%=\mathbf{2 , 1 8} \% \text {. }
$$

c) Albuminbestimmung.

Best. I. Best. II.

Filter und Albumin , 49,581 49,410

Filter . . . . $49,495 \quad 49,323$

Albumin $0,086 \quad 0,087$

$$
=0,42 \%=0,42 \% \text {. }
$$

c) Peptonbestimmung.

Verfahren und Normallösung wie oben.

$$
\begin{aligned}
& \text { Glas } a=58 \mathrm{cc}=\mathbf{0}, \mathbf{2 8} \% \text { Pepton. } \\
& \text { Glas } \mathrm{b}=\mathbf{5 6} \mathrm{co}=\mathbf{0}, \mathbf{2 7} \% \text { Pepton. }
\end{aligned}
$$

Die Peptonwerthe der beiden Methoden stimmen also auch in diesem Falle gut überein.

Versuch XII. 13/I 81. Versuchsmaterial: Zuletzt dem Euter entzogene Mittagmilch. Spez. Gew. 1,28. Die Milch wird zum Theil ganz frisch, zum Theil nach einer m. o. w. Tangen Finwirkung der Körperwärme untersucht. Hierbei wird das Pepton sowohl direkt als im Anschlusse an das Hoppe-Seyler'sche Verfahren bestimmt.

\section{A. Friche Milch.}

1) Direkte Peptonbestimmung.

40 cc Milch mit 40 cc Wasser versetzt, dann mit Kochsalz gesättigt 
und mit 80 cc der Essigsäure-Kochsalzmischung gefällt. Die weitere Behandlung wie oben angegeben. Die Normallösung 1: 1000.

Der gelöste Phosphorwolframsäureniederschlag stimmt bei 57 cc mit der Normallösung im Farbenton überein; d. h. also, die Milch besitzt einen Peptongehalt von $\mathbf{0 , 1 4} \%$.

2) Eiweiss- und Peptonbestimmung nach dem modif.

Hoppe-Seyler'schen Verfahren.

a) Caseïnbestimmung.

Filter und Caseïn . . 49,680

Filter . . . . . 49,147

Caseïn $\quad 0,533$

$$
=\mathbf{2 , 5 9} \%
$$

b) Albuminbestimmung.

Filter uud Albumin . . 49,248

Filter . . . . . . . 49,171

$$
\begin{aligned}
\text { Albumin } & 0,077 \\
= & \mathbf{0 , 3 7 \%} \% .
\end{aligned}
$$

c) Peptonbestimmung.

Bei 32 cc Uebereinstimmung mit der Normallösung; Peptongehalt demnach $0,15 \%$.

\section{B. Dieselbe Milch nach 3stündigem Digeriren bei $40^{\circ}$.}

1) Direkte Peptonbestimmung.

Verfahren wie oben. Bei 68 ce Uebereinstimmung mit der Normallösung. Peptongehalt also $\mathbf{0 , 1 6 \%} \%$.

2) Eiweiss - und Peptonbestimmung nach dem modif.

Hoppe-Seyler'schen Verfahren.

a) Caseinbestimmung.

Langsamen Filtrirens wegen werden zwei Filter benutzt.

$$
\begin{aligned}
& \text { Filter und Caseïn . . 49,381 49,462 } \\
& \text { Filter. . . . . . . } 49,187 \quad 49,141 \\
& \text { Caseïn } \underbrace{0,194 \quad 0,321}_{0,515} \\
& =\mathbf{2 , 5 0} \% \text {. }
\end{aligned}
$$

b) Albuminbestimmung.

Filter und Albumin . . 49,225

Filter . . . . . 49,150

$$
\begin{aligned}
& 0,075 \\
= & \mathbf{0 , 3 6} \% \text {. }
\end{aligned}
$$


c) Peptonbestimmung.

Bei 39 ce Uebereinstimmung mit dem Farbenton der Normallösung; Peptongehalt also $\mathbf{0 , 1 9} \%$.

\section{Dieselbe Milch nach 10stündigem Digeriren bei $40^{\circ}$.}

1) Direkte Peptonbestimmung.

Verfahren und Normallösung wie oben. Der gelöste Phosphorwolframsäureniederschlag stimmt bei 123 cc im Farbenton mit der Normallösung überein. Die Milch besitzt mithin einen Peptongehalt von $\mathbf{0 , 3 0 \%} \%$.

2) Eiweiss- und Peptonbestimmung nach dem modif.

Hoppe-Seyler'schen Verfahren.

a) Caseïnbestimmung.

Filter und Caseïn . . 49,345 49,454

Filter . . . . . . . $49,159 \quad 49,156$

$$
\text { Caseïn } \begin{gathered}
\frac{0,186 \cdot 0,298}{0,484} \\
=\mathbf{2 , 3 5} \% .
\end{gathered}
$$

b) Albuminbestimm ung.

Filter und Albumin . . 49,233

Filter. 49,155

$$
\begin{aligned}
& \text { Albumin } \quad 0,078 \\
& =\mathbf{0 , 3 8} \% \text {. }
\end{aligned}
$$

c) Peptonbestimmung.

Bei 57 ce Uebereinstimmung mit der Normallösung. Die Milch enthält also $\mathbf{0 , 2 8 \%} \%$ Pepton.

Versu ch XIIr. Morgenmilch, spez. Gew. 1,030. Ein Theil der Milch wird ganz frisch, ein anderer nach 6stïndigem Digeriren hei $40^{\circ}$ analysirt.

\section{A. Frische Mileh.}

a) Case inbestimmug.

Best. I. Best. II.

$$
\begin{array}{rrr}
\text { Filter and Caseïn . . } & 49,705 & 49,734 \\
\text { Filter . . . . . } & 49,161 & 49, \mathbf{1 8 7} \\
\hline \text { Caseïn } & 0,544 & 0,547 \\
& \mathbf{2 , 6 4} \% & \mathbf{2 , 6 5} \% .
\end{array}
$$

b) A I buminbestimmung.

Best. I. Best. II.

$$
\begin{aligned}
& \text { Filter und Albumin . . . 49,388 49,382 } \\
& \text { Filter . . . . . . . . 49,298 49,294 } \\
& 0,090 \quad 0,088 \\
& =0,44 \% \quad 0,43 \% \text {. }
\end{aligned}
$$


c) Peptonbestimmung.

Das caseïn- und albuminfreie Filtrat der Bestimmung I liefert auf Zusatz von Phosphorwolframsäure einen Niederschlag, der nach der Behandlung mit Natronlauge and Kupfervitriol bei 18 cc Uebereinstimmung mit einer $1 \% \%$ Normalpeptonlösung zeigt; das Filtrat der anderen Bestimmung misst unter den gleichen Verlälttnissen $17 \mathrm{cc}$.

Nach Bestimmung I enthält daher die Milch 0,09\% Pepton.

\section{B. Dieselbe Milch nach 6stündigem Digeriren bei $40^{\prime}$.}

a) Case ïnbestimmung.

Best. I. Best. II.

Filter und Caseïn . . . 49,698 49,658

Filter . . . . . . . 49,176 49,134

$$
\begin{aligned}
& \text { Caseïn } 0,522 \quad 0,524 \\
& =\mathbf{2 , 5 3} \%=\mathbf{2 , 5 4} \% \text {. }
\end{aligned}
$$

b) Albuminbestim $\mathrm{m}$ ung.

Best. I. Best. II.

Filter und Albumin . . 49,394 49,598

Filter . . . . . . . $49,304 \quad 49,508$

Albumin $0,090 \quad 0,090$

$$
=0,44 \%=0,44 \%
$$

c) Peptonbestimmung.

Bestimmuug I zeigt bei $28 \mathrm{cc}$, Bestimmung II bei 26 cc Uebereinstimmung mit der Normallösung. Die Milch enthält also 0,14, resp. $\mathbf{0 , 1 3} \%$ Pepton.

Versuch XIV. 28/X 81. Mittagmilch, spez. Gewicht 1,0306. Der Versuch bezweckt, die durch Estündiges Digeriren bei $40^{\circ}$, sowie die durch längeres Stehen bei gewöhnlicher Temperatur bewirkte Veränderung der Milch zu ermitteln.

\section{A. Frische Milch.}

a) Caseïnbestim ung.

Best. 1. Best. II.

$$
\begin{array}{rrr}
\text { Filter und Caseïn . , } 49,867 & 49,872 \\
\text { Filter . . . . . . . } 49,344 & 49,350 \\
\text { Casein } & 0,523 & 0,522 \\
& =\mathbf{2 , 5 4} \%=\mathbf{2 , 5 3} \% .
\end{array}
$$

b) Albuminbestim $\mathrm{mug}$.

Filter und Albumin. . 49,409 49,386

Filter . . . . . . 49,333 49,308

$$
\begin{array}{ccc}
\text { Albumin } & 0,076 & 0,078 \\
= & \mathbf{0 , 3 7 \%} \%=\mathbf{0}, \mathbf{3 8} \% .
\end{array}
$$


a) Peptoubestimmung.

Bestimmung I stimmt bei 25 ac mit dem Farbenton einer $1 \% \%$ Normallösung überein; Bestimmung II verunglückt. Die Milch enthält demnach $\mathbf{0 , 1 2} \%$ Pepton.

\section{B. Dieselbe Milch nach 8stündigem Digeriren bei $40^{\circ}$.}

a) Case in bestim $\mathrm{m}$ ng. Best. I. Best. II.

$$
\begin{aligned}
& \text { Filter und Caseïn . . 49,849 49,832 } \\
& \text { Filter. . . . . . 49,376 49,358 } \\
& \text { Caseïn } 0,473 \quad 0,474 \\
& =\mathbf{2}, \mathbf{2 9} \%=\mathbf{2 , 3 0} \% \text {. }
\end{aligned}
$$

b) Albuminbestimmug.

$$
\begin{array}{rrr}
\text { Filter and Albumin . } & 49,506 & 49,329 \\
\text { Filter. . . . . . } 49,430 & 49,254 \\
\text { Albumin } & \mathbf{0 , 0 7 6} & 0,075 \\
= & \mathbf{0 , 3 7} \%=\mathbf{0 3 7} \%
\end{array}
$$

c) Peptonbestimm ung.

Bestimmung I stimmt bei 55, Bestimmung II bei 57 ce mit der Normallösung überein; erstere zeigt demnach 0,27, letztere $0,28 \%$ Pepton an.

\section{Dieselbe Milch nach längerem Stehen bei Zimmerwärme.}

Eine Probe der Milch wird bis zur beginnenden Gerinnung (ca. 48 Stunden lang) bei $14^{\circ}$ stehen gelassen und alsdann analysirt.

a) Case inbestim mug.

2 ce der sehr verdünnten Essigsäure und ein starker Kohlensäurestrom genügen zur Ausfällung des Caseïns. Der Niederschlag ist äusserst locker. Best. I. Best. II.

$$
\begin{aligned}
& \text { Filter und Caseïn . . 49,821 49,785 } \\
& \text { Filter . . . . . . . 49,339 49,303 } \\
& \text { Caseïn } 0,482 \quad 0,482 \\
& =\mathbf{2}, \mathbf{3} 4 \%=\mathbf{2}, \mathbf{3 4} \% \text {. }
\end{aligned}
$$

b) A I buminbestimm ang.

Die Filtrate bleiben beim Aufkochen klar. Weiteres Zufügen von Essigsäure bewirkt nur geringe Trübung und erst beim Einengen auf ca. 50 bis 60 ce hat sich eine nennenswerthe Menge eines flockigen Niederschlages gebildet.

Best. I. Best. II.

$$
\begin{aligned}
& \text { Eilter und Albumin . 49,387 49,285 } \\
& \text { Filter . . . . . . 49,330 49,226 } \\
& \text { Albumin } \overline{0,057 \quad 0,059} \\
& =0,28 \%=0,29 \%
\end{aligned}
$$


c) Peptonbestimmung.

I. zeigt bei $49 \mathrm{cc}$, II bei 46 cc Uebereinstimmung mit der Normallösung. Die Milch enthält also 0,24, resp. 0,23\% Pepton.

Versuch XV. 5/IX 81. Der folgende Versuch zeigt die Veränderung der Milch durch 12stündiges Digeriren bei Körperwärme. Bemerkt sei, dass die zuerst aufgesammelte Milch getrennt von dem zuletzt gewonnenen Eutersekrete geprüft worden ist. Beide Hinterstriche lieferten zusammen etwa 3 Liter Milch. Hiervon wurden die ersten und letzten 500 cc gesondert aufgesammelt und analysirt.

\section{Erste Milch.}

Morgenmilch; spez. Gewicht 1,0338.

\section{A. Frische Milch.}

a) Case in bestimmung.

Best. I. Best. II.

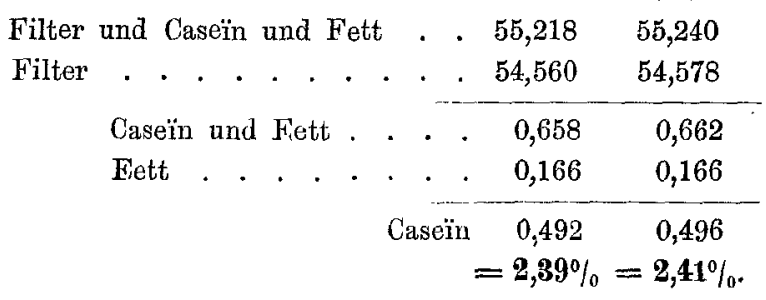

b) Albuminbestimmung.

Best. I. Best. II.

Filter und Albumin . 54,610 54,625

Filter. . . . . . $54,536 \quad 54,550$

Albumin $0,074 \quad 0,075$

c) Peptonbestimmung.

Bestimmung I stimmt bei $28 \mathrm{cc}$, II bei 29,5 cc mit der Normallösung überein; die Milch enthält demnach $\mathbf{0 , 1 4} \%$ Pepton.

B. Dieselbe Milch nach 12stündigem Digeriren bei $40^{\circ}$.

a) Case ïnbestimmung.

Best. I. Best. II.

Filter und Caseïn and Fett . . $55,199 \quad 55,143$

Filter . . . . . . . . . . $54,569 \quad 54,513$

Caseïn und Eett . . . . $0,630 \quad 0,630$

Eett . . . . . . . $0,166 \quad 0,166$

Caseïn $0,464 \quad 0,464$

$=2,25 \%=2.25 \%$.

E. Pflüger, Archiv f. Physiologie. Bd. XXVIIT. 
b) Albuminbestimmung.

$$
\text { Best. I. Best. II. }
$$

Filter und Albumin - $54,530 \quad 54,506$

Filter. . . . . . . $54,460 \quad 54,433$

$$
\begin{aligned}
& \text { Albumin } 0,070 \quad 0,073 \\
& =0,34 \%=0,36 \% \text {. }
\end{aligned}
$$

c) Peptonbestimm ang.

Bestimmung I stimmt bei 53 cc mit einer $1 \% \%$ Normallösung ïberein; Bestimmung II verunglückt. Die Milch würde demnach $\mathbf{0 , 2 6 \%}$ Pepton enthalten.

\section{Zuletzt gewonnene Milch.}

Spezifisches Gewicht: 1,0271.

\section{A. Frische Milch.}

a) Case inbestimmung.

Best. I. Best. II.

Filter und Caseïn . . 55,008 54,974

Filter. . . . . . . $54,552 \quad 54,514$

$$
\begin{array}{ccc}
\text { Caseïn } & 0,456 & 0,460 \\
=\mathbf{2 , 2} & \mathbf{2 1} \%=\mathbf{2 , 2 3} \% .
\end{array}
$$

b) Albuminbestimmung.

Best. I, Best. II.

Filter und Albumin . $54,614 \quad 54,594$

Filter . . . . . . . $54,536 \quad 54,512$

Casein $\begin{array}{rc}0,078 & 0,082 \\ = & \mathbf{0 , 3 8} \%=\mathbf{0 , 4 0} \% .\end{array}$

c) Pepton bestimm ung.

I stimmt bei 42 , II bei 44 cc mit der Normallösung überein. Die Milch enthält also $\mathbf{0 , 2 0}$, resp. $\mathbf{0 , 2 1 \%}$ Pepton.

B. Dieselbe Milch nach 12stindigem Digeriren bei $40^{\circ}$.

a) Casein bestimm ung.

$$
\text { Best. I. Best. II. }
$$

Filter und Caseïn . . 54,777 54,798

Filter . . . . . . $54,373 \quad 54,396$

Caseïn $0,404 \quad 0,402$

$$
=1,96 \%=1,95 \% \text {. }
$$

b) A 1 buminbestimmug.

Best. I. Best. II.

Filter und Albumin . 54,582 54,518

Filter.

$$
\begin{array}{ccc}
. & 54,500 & \mathbf{5 4 , 4 4 0} \\
\text { Albumin } & 0,082 & 0,078 \\
= & \mathbf{0 , 4 0} \%=\mathbf{0 , 3 8} \% .
\end{array}
$$




$$
\begin{aligned}
& \text { c) Peptonbestim m ung. } \\
& I=70,0 \text { cc }=\mathbf{0 , 3 4} \% \text { Pepton. } \\
& I I=72,0 \text { co }=\mathbf{0 , 3 5} \%
\end{aligned}
$$

Versuch XVI. 15/IX 81. Norgenmilch, und zwar die dem Euter zuerst entzogenen 500 cc aus beiden Hinterstrichen. Spez. Gewicht 1,0325. Ein Theil der Milch wird frisch, der andere nach 24 stïndigem Digeriren bei $40^{\circ}$ analysirt.

\section{A. Frische Milch.}

a) Case inbestim $m u n g$.

Best. I. Best. II.

$$
\begin{aligned}
& \text { Filter und Caseïn und Fett . . 55,056 55,084 } \\
& \text { Filter . . . . . . . . . . } 54,468 \quad 54,502 \\
& \text { Casein und Fett . . . . } 0,588 \quad 0,582 \\
& \text { Fett . . . . . . . } 0,124 \quad 0,124 \\
& \text { Caseïn } 0,464 \quad 0,458 \\
& =2,25 \%=2,22 \% \text {. }
\end{aligned}
$$

b) Albuminbestimmung.

Best. I. Best. II.

$$
\begin{aligned}
& \text { Filter und Albumin . } 54.519 \quad 54,520 \\
& \text { Filter . . . . . } 54,455 \quad 54,460 \\
& \text { Albumin } 0,064 \quad 0,060 \\
& =0,31 \%=0,29 \% \text {. }
\end{aligned}
$$

c) Peptonbestimmung.

Bestimmung $\mathrm{I}=27 \mathrm{cc}=\mathbf{0 , 1 3} \%$ Pepton.

$" \quad$ II $=29 \mathrm{cc}=\mathbf{0}, \mathbf{1 4} \% \quad$ "

B. Dieselbe- Milch nach 24 stïndigem Digeriren bei $40^{\circ}$.

b) Caseïnbestimmung.

Best. I. Best. II.

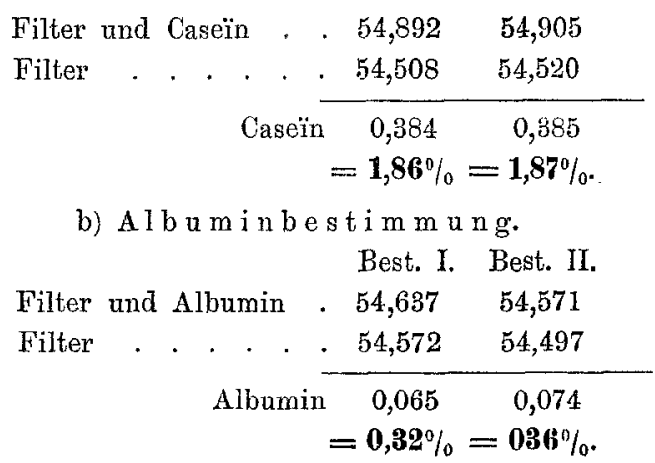




$$
\begin{aligned}
& \text { c) Peptonbestimmung. } \\
& \text { Bestimmung } \mathrm{I}=70 \mathrm{cc}=\mathbf{0 , 3 4} \% \text { Pepton. } \\
& \Rightarrow \quad \mathrm{II}=65,5 \mathrm{cc}=\mathbf{0 , 3 2} \%
\end{aligned}
$$

Durch die bisherigen Versuche ist also festgestellt, dass die frische Milch einen ganz nennenswerthen Peptongehalt besitzt, der sich durch Digeriren bei Körperwärme oder durch längeres Stehen bei gewöhnlicher Temperatur derartig vermehrt, dass die Menge des Peptons jetzt derjenigen des Albumins nahe kommen kann, und weiter, dass das Casein als die Muttersubstanz des Peptons betrachtet werden muss.

Ob neben dem Pepton noch andere Körper aus dem Caseïn hervorgehen, ist mir bei der Kürze der Zeit, die mir noch zur Beendigung der Arbeit zur Verfügung stand, nicht bekannt geworden. Stellt man die Caseïnabnahme in Vergleich mit der Peptonzunahme, so wird man stets einen bemerkenswerthen Ausfall finden und es gewinnt in der That den Ansehein, dass das Pepton nicht das einzige Umwandlungsproduckt des Caseïns sei; es betrug nämlich die

\begin{tabular}{c|c}
\hline Caseïnabnahme. & Peptonzunahme. \\
\hline $0,09 \mathrm{gr}$ & $0,04 \mathrm{gr}$ \\
$0,11 "$ & $0,05 \mathrm{~g} "$ \\
$0,20 "$ & $0,16 "$ \\
$0,24 "$ & $0,13 "$ \\
$0,24 "$ & $0,15 "$ \\
$0,26 "$ & $0,16 "$ \\
$0,37 "$ & $0,21 "$
\end{tabular}

\section{Nähere Entstehungsweise des Peptons.}

Bei den Kenntnissen, die wir von der Peptonisirung des Caseïns überhaupt besitzen, lag es nahe, den Mehrgehalt an Pepton in der digerirten Milch auf die Thätigkeit von Fermentkräften zurtickzuführen. Sollten letztere wirklich im Spiele sein, so musste die Peptonbildung bei einer Milch, auf welche unmittelbar vor dem Digeriren die Siedhitze eingewirkt hatte, in Wegfall kommen. Das trifft nun, wie nachfolgender Versuch beweist, in der That zu:

Versuch XVII. Von der im Versuch XIII benutzten Milch wird ein Theil sogleich nach dem Melken gekocht und alsdann 6 Stunden hindurch bei $40^{\circ}$ digerirt. Die sodann vorgenommene Analyse ergab folgende Werthe: 


\section{a) Caseinbestimmung.}

Die nachfolgenden Werthe sind nicht allein auf Caseïn zurückzuführen, sondern sie umschliessen auch den grössten Theil des Albumins, welches, wie bekannt, durch Einwirkung der Siedhitze zur Ausfällung gelangt.

Best. I. Best. II.

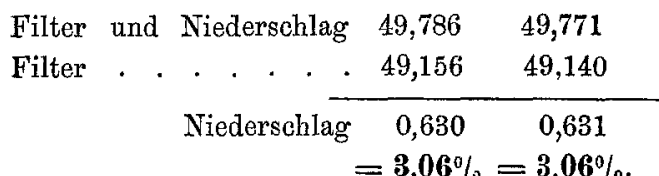

b) Albuminbestim mu g.

Beim Einengen der Filtrate auf kleine Volumina scheiden sich nur geringe Mengen eines flockigen Niederschlages aus.

Best. I. Best. II.

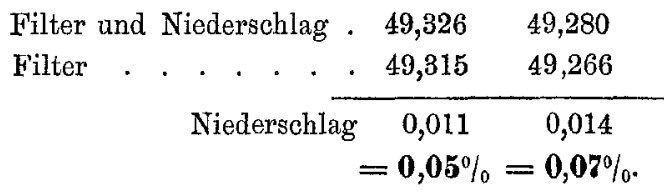

c) Peptonbestimmung.

Der gelöste und mit Kupfer versetzte Phosphorwolframsäureniederschlag der Bestimmung I nimmt ein Volumen von $16 \mathrm{cc}$ ein, als sein Farbenton mit demjenigen einer $1 \% \%$ Normalpeptonlösung übereinstimmt, während derjenige der Bestimmung II unter den gleichen Umständen 17 co misst. Die digerirte Milch enthält daher 0,08\% Pepton. Da der Peptongehalt der ganz frischen Milch gleichfalls $\mathbf{0 , 0 8} \%$ betrug (s. p. 301), so hat demnach die gekochte Milch durch 6 stïndiges Digeriren bei $40^{\circ} \mathrm{keine}$ Peptonzun a h m e erfahren.

Da nun die Milch auch beim Stehen bei gewöhnlicher Temperatur fortwährend an Pepton zunimmt (s. Versuch XIV), so war die Möglichkeit nicht abzuweisen, dass die Peptonzunahme mit der Thätigkeit des Milchsäurefermentes in Verbindung stehe; indessen verliert diese Annahme durch den Umstand ihre Berechtigung, dass es nicht gelingen wollte, in frisch gekochter und abgekühlter Milch durch Zusatz von Milchsäureferment eine Caseïnabnahme zu erzielen (Versuch XVIII). Auch wurde die Caseïnzerstörung durch Zufügen von Salicyl- oder Carbolsäure zur Milch durehaus nicht aufgehalten (Versuch XVIII und XIX). 
Versuch XVIII. Morgenmileh, spez. Gew. 1,0312.

\section{A. Ganz frische Vilch.}

a) Case in bestim $\mathrm{m}$ ung.

Best. I. Best. II

\begin{tabular}{ccc} 
Filter und Caseïn . . 49,688 & 49,687 \\
Filter . . . . . . . 49,170 & 49,170 \\
\hline & \multicolumn{4}{c}{ Caseïn } & 0,518 & 0,517 \\
& $=\mathbf{2 , 5 1 \%} \%=\mathbf{2 , 5 1} \%$.
\end{tabular}

b) Albuminbestimmung.

Filter und Albumin . 49,362 49,453

Filter . . . . . . $49,273 \quad 49,364$

$$
\begin{aligned}
& \text { Albumin } \quad 0,089 \quad 0,089 \\
& =0,43 \%=0,43 \%_{0} \text {. }
\end{aligned}
$$

B. Dieselbe Milch, gekocht, nach dem Abkïhlen mit 2 Tropfen Serum von spontan geronnener Milch versetzt und alsdaun 6 Stunden

\section{hiudurch bei $40^{\circ}$ digerirt.}

Best. I. Best. II.

Filter und Caseïn und durch Sied-

\begin{tabular}{rcc} 
hitze gefälltes Albumin & 49,709 & 49,749 \\
Filter. . . . . . . 49,128 & 49,160 \\
\cline { 2 - 3 } Casein und Albumin & 0,581 & 0,589 \\
$=\mathbf{2 , 8 2} \%=\mathbf{8 , 8 6} \%$.
\end{tabular}

Da nun beim Kochen der Milch nach meinen Erfahrungen durchschnittlich nock 0,06-0,08\% Albumin. ungefällt bleiben, so ergibt sich, dass das Digeriren bei Gegenwart von Milchsäureferment keine Caseïnabnahme bewirkt hat.

C. Dieselbe Milch mit soviel Carbolsänre versetzt, dass der Gehalt daran $0,5 \%$ ausmacht und alsdann 6 Stunden hindurch bei $40^{\circ}$ digerirt.

a) Caseïnbestimmung.

$$
\text { Best. I. Best. II. }
$$

$$
\begin{aligned}
& \text { Filter und Caseïn . . 49,598 49,599 } \\
& \text { Filter . . . . . . . 49,132 49,132 }
\end{aligned}
$$

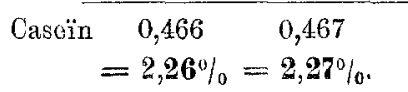

b) Albuminbestimm ung.

$$
\text { Best. I. Best. II. }
$$

Filter und Albumin . 49,225 49,234:

Filter. . . . . . $49,125 \quad 49,14 \%$

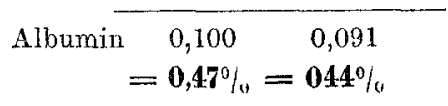


Ungeachtet des recht bedeutenden Carbolsäurezusatzes ist also eine sehr erhebliche Caseïneinbusse zu konstatiren.

Versuch XIX. Frische Milch mit einem Caseïngehalte von $2,64 \%$ und einem Albumingehalte von $0,44 \%$ (vergl. Versuch XIII) wird mit soviel Salicylsäure versetzt, dass der Gehalt an dieser Substanz $1 \% \%$ beträgt. Alsdann wird die Milch 6 Stunden hindurch bei $40^{\circ}$ digerirt, während eine andere Probe ohne Salicylsäure genau ebenso behandelt wird.

a) Case inbestim $\mathrm{m} u \mathrm{~g}$.

Mit Salicyls. vers. Milch.| Einf. diger. Milch. Best. I. Best II. Best. I. Best. II.

\begin{tabular}{lll|rr} 
Filter und Caseïn . 49,725 & 49,690 & $49,698 \quad 49,658$
\end{tabular}
Filter. . . . . . $49,203 \quad 49,164 \quad 49,176 \quad 49,135$

Caseïn \begin{tabular}{rr|cc}
0,522 & 0,526 & 0,522 & 0,523 \\
$=\mathbf{2 , 5 3} \%$ & $=\mathbf{2 , 5 5} \%$ & $=\mathbf{2 , 5 3} \%$ & $=\mathbf{2 , 5 4} \%$
\end{tabular}

b) A 1 buminbestimmug.

Mit Salicyls. vers. Milch.: Einf. diger. Milch.

\begin{tabular}{lrc|rc} 
& Best. I. & Bsst. Il. & Best. I. & Best. II. \\
Filter und Albumin & 49,269 & 49,243 & 49,394 & 49,598 \\
Filter . . . . . . . & 49,180 & 49,152 & 49,304 & 49,508 \\
\hline Albumin & 0,089 & 0,091 & 0,090 & 0,090 \\
$=\mathbf{0 , 4 3} \%=\mathbf{0 , 4 4} \%$ & $=\mathbf{0 4 4} \%=\mathbf{0 4 4} \%$.
\end{tabular}

In beiden Proben hat also die Caseïnabnahme in demselben Umfange stattgefunden.

Sprachen somit die bisherigen Beobachtungen dafür, dass die Caseïnabnahme an das Vorhandensein eines peptonbildenden Fermentes geknüpft sei, so musste der Umstand, dass es nicht gelingen wollte, die Wirksamkeit dieses Fermentes durch Antiseptica zu hemmen, auf eine grosse Aehnlichkeit desselben mit den eiweissverdauenden Fermenten hinweisen.

Da nun Brii cke angiebt, dass das Pepsin im unveränderten Zustande resorbirt werde und zur Ausscheidung gelange (so z. B. vermochte Brïcke im Nierensekrete Pepsin nachzuweisen), so war die Möglichkeit nicht von der Hand zu weisen, dass auch unsere Erscheinung auf eine Pepsinwirkung zuriuckzuführen sei.

Dieser Punkt schien einer experimentellen Prufung durchaus nicht unzugänglich. Kühne bereits gibt an, dass Pepsin durch Einwirkung von Natronlauge zerstört wird und ich fand, dass dieser Erfolg schon mit völliger Sicherheit za erreichen ist, wenn die Pepsinlösung nur $1 / 10000$ freies Natron enthält. Wäre daher das 
peptonbildende Ferment, thatsächlich Pepsin, so mïsste die Caseïnabnahme bei Anwendung einer mit Natronlauge alkalisch gemachten und dann mit Phosphorsäure bis zur schwach sauren Reaktion versetzten Milch vollständig in Wegfall kommen. Das ist aber, wie der folgende Versuch darthut, nicht der Fall.

Vers u ch XX. Milch mit einem Caseïngehalte von 2,59\%, einem Albumingehalte von $0,27 \%$ und einem Peptongehalte von $0,14 \%$ (vergl. Versuch XII) wird mittels Natronlauge deutlich alkalisch gemacht, nach kurzer Zeit mittels Phosphorsäure ganz schwach angesäuert und alsdann 10 Stunden hindurch bei $40^{\circ}$ digerirt. Die nunmehr vorgenommene Analyse ergab:

a) Caseinbestimmung.

$$
\begin{aligned}
& \text { Filter und Caseïn . . . , , 49,704 } \\
& \text { Filter . . . . . . . . . 49,208 } \\
& \text { Caseïn } \quad 0,496 \\
& =\mathbf{2}, \mathbf{4 1} \% \text {. }
\end{aligned}
$$

b) Albuminbestim ang.

Filter and Albumin. . . . . 49,244

Filter. . . . . . . . : 49,169

$$
\begin{aligned}
\text { Albumin } & 0,075 \\
= & \mathbf{0 , 3 6} \% .
\end{aligned}
$$

c) Peptonbestimmung.

Die Milch enthält $\mathbf{0 , 2 3} \%$ Pepton.

Weiteren Bestrebungen nach der Aufdeckung des in der Milch enthaltenen peptonbildenden Fermentes setzte meine Abreise von Proskau ein Ziel, was ich, namentlich auch mit Riicksicht auf die Bedeutung, welche das aufgefundene Verhalten des Caseïns für die Praxis der Käserei besitzen muss, lebhaft bedauere.

\section{Ergebis se.}

Aus den mitgetheilten Versuchen dürfte Folgendes mit Sicherheit hervorgehen:

1) In der Milch sind mit Hülfe geeigneter Methoden regelmässig drei eiweissartige Körper aufzufinden:

a) Caseïn,

b) Albumin,

c) Pepton.

2) Der Gehalt an Caseîn betrug in der frischen Milch im Durchschnitt von 7 Versuchen $2,43 \%$ und be- 
wegte sich innerhalb der Grenzen von $2,21-2,64 \%$; der Albumingehalt stellte sich bei der gleichen Anzahl von Versuchen im Durchschnitt auf $0,38 \%$ und schwankte zwischen 0,29 und $0,44 \%$; der mittlere Peptongehalt machte $0,13 \%$ aus, während der für Pepton ermittelte Werth überhaupt in den Grenzenvon 0,08 and $0,19 \%$ lag.

3) In Folge eines Digerirens der Milch bei Körperwärme erleidet das Caseïn eine merkliche Einbusse, während das Pepton unter den gleichen Verhältnissen eine nennenswerthe Zunahme erfährt. Bei Einwirkung der gewöhnlichen Zimmerwärme findet dieselbe Veränderung, jedoch weit langsamer statt. Der Umfang dieser Zu - und Abnahme zeigt sich der Dauer des Digerirens proportional.

4) Der Peptongehalt kann derartig anwachsen, dass er dem Albumingehalt an Grösse fast gleichkommt. In einem Falle stieg er auf $0,33 \%$, während der Albumingehalt $0,34 \%$ ausmachte.

5) Das Pepton geht durch einen fermentativen Umwandlungsprozess aus dem Caseïn hervor und scheint nicht das einzige Produkt desselben zu sein, wenigstens zeigte sich die Peptonzunahme stets merklich geringer als die Caseïnabnahme.

6) Das Ferment wird dureh Siedhitze zerstört, büsst aber dureh angemessenen Zusatz von Salicyl-und Carbolsäure seine Wirksamkeit nicht ein und erinnert in diesem Verhalten an die eiweissverdauenden Fermente. Ein Nachweis, dass es mit Pepsin identisch sei, woll te nicht gelingen.

7) Während das fertige Drüsensekret keineswegs einen gleichbleibenden Caseïn- und Peptongehalt besitzt, die hierfür gef undenen Werthe vielmehr von der mehr oder weniger frischen Beschaffenheit der Milch abhängig sind, zeigt sich der Albumingehalt weit konstanter. Er erleidet durch Digeriren bei Körperwärme keine erkennbare Einbusse und beim Stehenlassen der Milch bis zum Eintritt der Gerinn ung ist die Abnahme auch nur eine geringe. 
8) Der Nachweis, dass die Milch darch längeres Stehen eine grosse Einbusse an Case in ( $10 \%$ und mehr) erleiden kann, dürfte für die milchwirthschaftliche Technik von boher Bedeutung sein und es dürfte als rationell erscheinen, die Milch in einem möglichst frischen Zustande zu verkäsen. Bei Anfertigung von Magerkäse dürfte dasjenige Aufrahmungsverfahren das beste sein, welches unter sonst gleichen Umständen die geringste Zersetzung des Caseins im Gefolge hat.

Am Schlusse bemerke ich noch, dass mir auch bei Ausführung dieser Arbeit die Hülfsmittel des milchwirthschaftlichen Institutes zu Proskau zur Verfügung standen.

Beiträge zur Kenntniss der Innervation des Herzens. Dritte Mittheilung.

VI. Die Deatung einiger Giftwirkangen am Froschherzen. Von

\section{Dr. M. L $\ddot{\mathbf{o}} \mathbf{w} \mathbf{i t}$,}

Privatdocent und Assistent am Institut für experimentelle Pathologie in Prag.

Hierzu Tafel II.

Die Annabme mehrerer verschieden functionirender nervöser Centralapparate im Herzen (speziell im Frosehherzen) wurde hauptsächlich auf Grund pharmakologischer Untersuchungen gemacht. Trotz der reichhaltigen Literatur, die gerade über diesen Gegenstand schon aufgespeichert wurde, ist aber das Verständniss, das man durch die hier einschlägigen Untersuchungen für den Innervationsvorgang des Herzens zu gewinnen trachtete, durchaus nicht erleichtert worden. 